\title{
GPPS-BJ-2019-0222
}

\section{Flow instabilities detection in centrifugal blower using empirical mode decomposition}

\author{
Mateusz Stajuda \\ Lodz University of Technology \\ mateusz.stajuda@p.lodz.pl \\ Lodz, Poland
}

\author{
Grzegorz Liśkiewicz \\ Lodz University of Technology \\ grzegorz.liskiewicz@p.lodz.pl \\ Lodz, Poland
}

\author{
David Garcia \\ University of Edinburgh \\ david.garcia@ed.ac.uk \\ Edinburgh, United Kingdom
}

\begin{abstract}
The aim of this article is to present the application of empirical mode decomposition (EMD) for centrifugal blower flow instabilities detection. The analysis of pressure signal features extracted by EMD technique provides indicators of flow phenomena, which could be used for creating an efficient data-based controller. Quasi-dynamic pressure signals from industrial-size blower are used as an input data for EMD algorithms. An energy-based approach to intrinsic mode functions (IMF) is applied, showing the possibility of condition monitoring and instabilities detection, distinctly displaying surge conditions and inlet recirculation. Different intrinsic mode functions (IMFs) are used to detect different instabilities. EMD also presents some potential in detection of optimal operation conditions for impeller, providing additional benefit for a control system. The possibilities of EMD analysis applied to centrifugal blowers and compressors will be further investigated.
\end{abstract}

\section{INTRODUCTION}

Aerodynamic instabilities detection and avoidance in compressors has been a topic of interest for numerous researchers for over 75 years (Day 2015). Surge and stall are major phenomena influencing compressor performance and posing a threat to machine operation. Surge presents itself by low frequency fluid oscillations at characteristic system frequency, which is often close to the Helmholtz frequency. The structure vibration it induces are dangerous to the machine operation and can cause its destruction (Fink et al. 2008; Liskiewicz and Horodko 2015)

Surge detection in centrifugal compressors is often based on previously created compressor map and locating machine operating position on it in continuous manner. The surge line is found for a particular compressor and certain margin - surge margin is created, moving the possible compressor operating point away from this line (Kurz et al. 2018). This approach, although safe and widely used, decreases compressor operating range and sometimes disallows the machine to work in the highest point of its performance curve (Fig. 1). Thus, a method allowing a compressor to safely operate close to the surge is desired. Such a goal could be attained by decreasing surge margin on compressor map, which will undoubtedly decrease safety. Other approach could be a different surge detection method, not based on a compressor map.

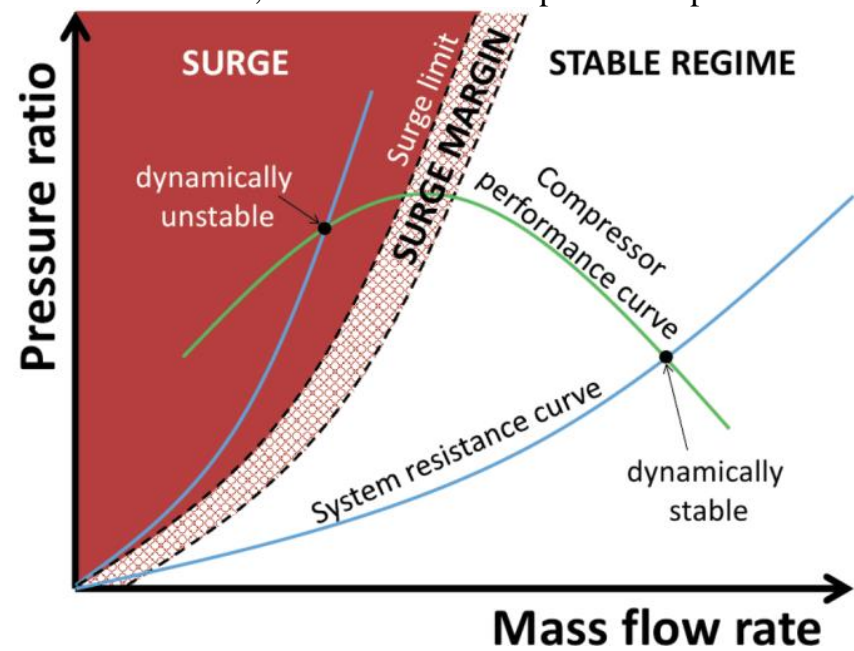

Figure 1. Centrifugal compressor performance map (Garcia and Liśkiewicz 2016)

Data-driven fault detection techniques for rotating machinery have been extensively used in many branches of industry. By means of time-series analysis, anomalies in the signal can be detected, indicating a presence of some type of 
fault. One of the highly employed fault extraction methods is empirical mode decomposition (EMD). Developed in 1998 by Huang (Huang et al. 1998), EMD is adaptive to signal, with a posteriori defined decomposition base, making it a powerful tool for nonlinear and non-stationary processes analysis.

Successfully used for many detection cases in different machines, EMD was capable of detecting bearings faults (Yu et al. 2005; Li et al. 2009), gear flaws (Fan and Zuo 2008; Singh and Zhao 2016) or shaft defects (Bin et al. 2012). The extent and number of valuable research using EMD is very wide (Lei et al. 2013).

Surprisingly, application of EMD to compressor instability detection has been limited, despite demonstrated utility of this method. Li et al. (Li et al. 2011) used EMD to detect surge in axial compressor. They focused on frequency domain, showing that EMD can effectively find changes in frequency band of interest, indicating instability. $\mathrm{Wu}(\mathrm{Wu}$ et al. 2016) has shown that EMD has a potential for the surge detection and can do it faster than the wavelet transform-based methods. Wu's approach used maximum amplitude of a certain intrinsic mode function (IMF), being a decomposition product. By a rise of amplitude, it was possible to detect the oncoming surge.

Changes of flow stability in a centrifugal machine produce pressure oscillations of varying frequency, allowing to determine machine condition (Liskiewicz and Horodko 2015). The EMD method might provide a suitable tool for the analysis of changes in the signal and quantification of machine flow conditions.

The aim of this article is to validate the applicability of empirical mode decomposition and IMF energy changes for detection of a centrifugal blower flow instabilities.

\section{METHODOLOGY}

\section{Experimental investigation}

Experimental investigation was performed on single stage centrifugal blower DP1.12, described in details by Liśkiewicz (Liśkiewicz 2014). The test stand was equipped with five subminiature Kulite transducers, mounted flush to the shroud walls to measure the static pressure. Their positions are presented in Fig. 2: $\mathrm{ps}_{\text {in }}$ - inlet static pressure, $\mathrm{ps}_{\text {out }}$ - outlet static pressure, $\mathrm{ps}_{\mathrm{imp} 1}$ - static pressure at the impeller shroud (0.2L), $\mathrm{ps}_{\text {imp2 }}$ - static pressure at the impeller shroud $(0.4 \mathrm{~L})$, $\mathrm{ps}_{\text {imp3 }}$ - static pressure at the impeller shroud $(0.9 \mathrm{~L})$. The value of $\mathrm{L}$ corresponded to the position along the shroud, where $\mathrm{L}=0$ is at the blade leading edge, $\mathrm{L}=1$ at trailing edge.

Blower design point was attained at nominal rotation frequency from $=120 \mathrm{~Hz}$, nominal mass flow $\mathrm{m}_{\text {nom }}=0.8$ and nominal pressure ratio ${ }_{\text {nom }}=1.12$ for ambient inlet conditions. In the experiment blower worked at lower conditions of rotational frequency $f_{\text {rot }}=100 \mathrm{~Hz}$, mass flow $\mathrm{m}^{\circ}=0.75 \mathrm{~kg} / \mathrm{s}$ and pressure ratio $=1.08$ due to damage risk at surge. Then, it was closest to its design point for throttle opening area of $30 \%$.

Data were acquired through quasi-dynamic measurements, aimed at examination of pressure oscillations and its dependency on throttling. Measurements were conducted for several valve positions, described by the dimensionless throttle opening area parameter (TOA). Its value described the percentage of the valve opening area.

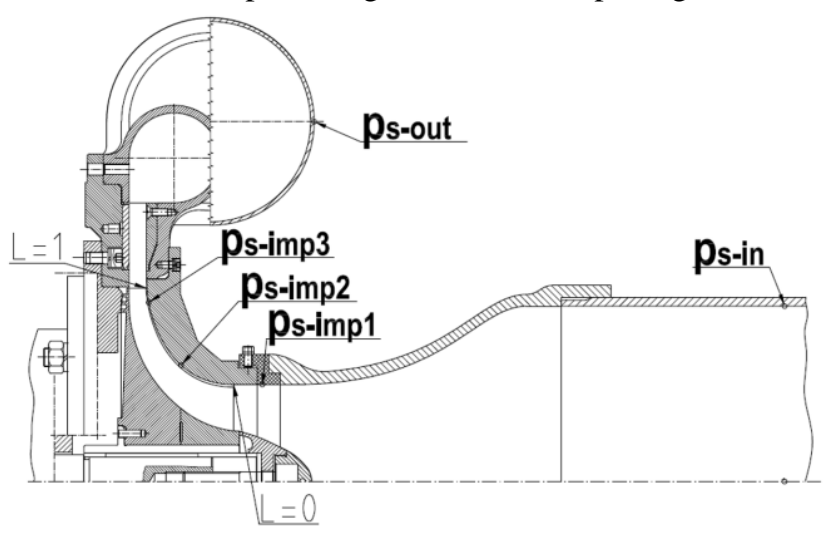

Figure 2. Experimental stand cross-section with sensors positions (Liskiewicz and Horodko 2015)

The data used in this investigation were obtained and previously analyzed by Liśkiewicz (Liskiewicz and Horodko 2015) using discrete wavelet transform. The centrifugal blower was operating with varying outlet valve closure, replicating the receiver resistance. Quasi-dynamic spectral maps were created, allowing to identify where the machine works in a stable manner and where the instabilities arise and start to dominate (Liśkiewicz et al. 2014).

For described investigation, a series of data for different throttle opening areas (TOA) was considered. TOA values ranged from $8.5 \%$, where deep surge was present, to $33 \%$, where the machine was working in a stable regime. In between those values, the flow transformed from stable to unstable. In the process, certain local instabilities could be observed, such as inlet recirculation. The analysis of this phenomenon was done by Garcia (Garcia et al. 2015). Table 1. Summarizes TOA values subjected to analysis and observed flow conditions.

Table 1. Throttle opening area and observed flow conditions (Liśkiewicz et al. 2014).

\begin{tabular}{|c|c|}
\hline TOA [\%] & Flow conditions \\
\hline 8.5 & Unstable - Deep surge \\
\hline 12 & Unstable - Mild surge \\
\hline $19-25$ & Transient - Inlet recirculation \\
\hline $26-33$ & Stable \\
\hline
\end{tabular}

\section{Decomposition procedure}

The empirical mode decomposition method is able to deal with nonstationary and nonlinear data of different origin. It is direct and adaptive, with a decomposition base derived from the data. The empirical mode decomposition method assumes that any signal consists of different simple intrinsic modes (IMFs) of oscillations, which can be identified (Huang et al. 1998). IMFs, due to their derivation, can reflect changes in both amplitude and frequency of phenomena in analyzed signal. For an extracted function to be termed an IMF, it must meet 2 conditions (Huang 2014):

- It must have the same (or differing by one) number of extrema and zero crossings

- The mean value of envelope defined by local maxima and minima must be equal to zero. 
The process of obtaining intrinsic mode functions is called sifting. To create IMFs from signal, a signal envelope must be obtained. To do so, all the local maxima must be identified and connected by cubic spline, creating the upper envelope. Then, the procedure should be repeated for the local minima to obtain the lower envelope. The upper and lower envelopes should cover all the data between them. The first component of decomposition $\boldsymbol{h}_{1}$ is obtained from (1), where $\boldsymbol{x}(t)$ is original data vector containing series of measurements dependent on time $t$ and $\boldsymbol{m}_{1}$ is the envelope mean.

$$
\boldsymbol{h}_{1}(\mathrm{t})=\boldsymbol{x}(t)-\boldsymbol{m}_{1}(t)
$$

Then, it is checked if $\boldsymbol{h}_{1}$ meets the conditions of an IMF. If not, then next process step takes place. The $\boldsymbol{h}_{1}$ is treated as an input data and $\boldsymbol{h}_{2}$ is created by subtracting the mean of $\boldsymbol{h}_{1}$ envelope from itself.

$$
\boldsymbol{h}_{2}(\mathrm{t})=\boldsymbol{h}_{1}(\mathrm{t})-\boldsymbol{m}_{2}(t)
$$

The process is repeated until the function meets IMF criteria or stoppage condition. In this research, a Cauchy type convergence test was used (Huang et al. 1998), with 0.2 limit. Assuming process is repeated $n$ times, $\boldsymbol{h}_{n}$ is designated $\boldsymbol{c}_{1}$ and becomes first IMF (marked further IMF 1). To continue the sifting, $\boldsymbol{c}_{1}$ is extracted from the original data $\boldsymbol{x}(t)$, the output of this operation is termed residue $\boldsymbol{r}_{1}(t)$.

$$
\boldsymbol{r}_{1}(t)=\boldsymbol{x}(t)-\boldsymbol{c}_{1}(t)
$$

The residue $\boldsymbol{r}_{1}(t)$ is taken as the original data and the sifting process is repeated, until the final residue is either a constant or a monotonic function. The signal after decomposition is divided into $k$ intrinsic mode functions $\boldsymbol{c}_{1}(t)$ to $\boldsymbol{c}_{k}(t)$ and a final

residue, $\boldsymbol{r}_{k}(t)$ containing information about trend in the data.

$$
\boldsymbol{x}(t)=\sum_{i=1}^{k} \boldsymbol{c}_{i}(t)+\boldsymbol{r}_{k}(t)
$$

The pressure signals from blower were subjected to the empirical mode decomposition. The analysis of intrinsic mode function can be done in various ways, relying on IMF amplitude (Wu et al. 2016) or IMFs subjected to Hilbert transform (Yu et al. 2005; Goharrizi and Sepehri 2012). Due to source of data and signal character, an energy-based approach was adopted for blower data. For each IMF, a root mean squared value was calculated according to (5), where $\boldsymbol{c}_{i}(m)$ is an $\mathrm{m}$-th value of $\mathrm{i}$-th IMF vector.

$$
\boldsymbol{R} \boldsymbol{M} \boldsymbol{S}_{I M F i}=\sqrt{\frac{1}{M} \sum_{m=1}^{M}\left|\boldsymbol{c}_{i}(m)\right|^{2}}
$$

The analysis of data was done in two steps. First, decomposition was performed on signals having different length. They were obtained by dividing a signal ( 20 seconds) for a particular TOA and sensor into several portions. The IMF RMS value for subsequent pieces was computed to establish a variability of IMF RMS value for a different signal length. It was done to ensure that a random signal portion taken from the whole data is representative for a particular TOA.

\section{RESULTS AND DISCUSSION}

\section{IMF stability and frequency spectra}

To establish the behavior of IMFs for different input, their stability for different signal length was examined.
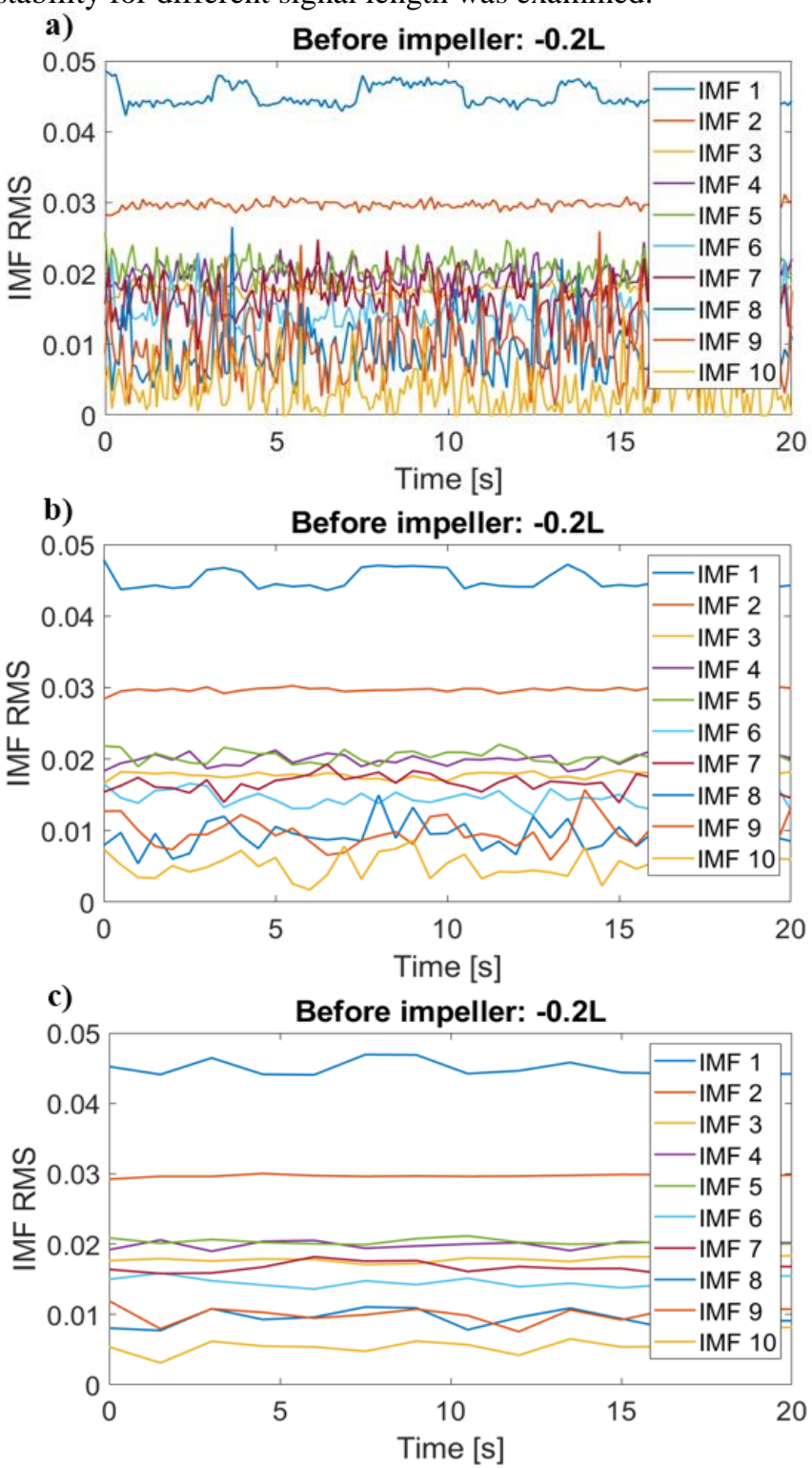

d)

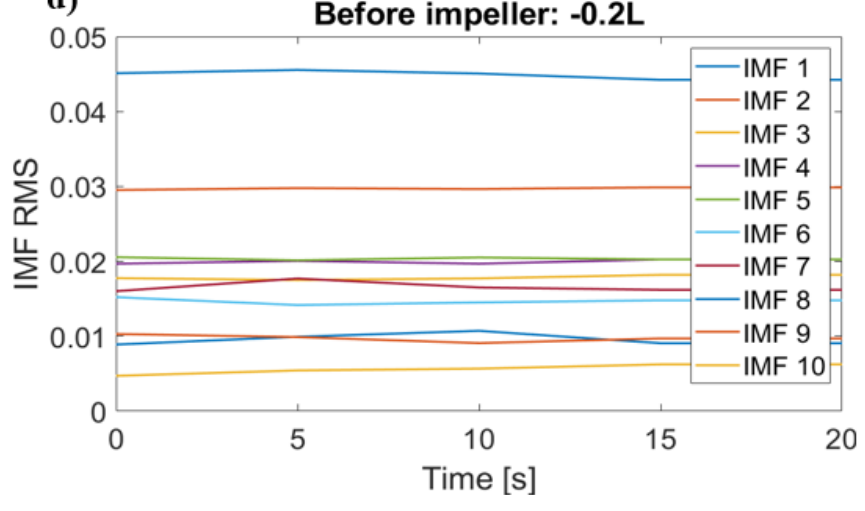

Figure 3. IMF RMS in time for different signal length at stable conditions TOA = 30\%; a) 10000 samples per point; b) 50000 samples per point; c) 150000 samples per point; d) $\mathbf{5 0 0} 000$ samples per point 
Four windows were used on signal sampled with frequency of $100 \mathrm{kHz}$. Shortest window incorporated 10000 samples, equivalent to 10 impeller revolutions $(0.1 \mathrm{~s})$. Second took 50000 samples, third 150000 samples and longest used 500000 samples per point, equivalent to 500 impeller revolutions $(5 \mathrm{~s})$. Input data was taken at stable conditions $(\mathrm{TOA}=30 \%)$ for a sensor located upstream of the impeller.

Signal length providing the best stability to length ratio was chosen for further analysis. Then, for this signal length, the value of IMF RMS was compared at different TOA, showing changes in the intensity of components fluctuations, which provided indication of flow conditions.

In terms of EMD applicability for dynamic controller design, the considered signal should be sufficiently short to decrease computational requirements and response time coming from necessary data collection period. Unfortunately, pressure signal collected at a high rate incorporates significant portion of noise and turbulence-related randomness. Therefore a longer collection period might enable avoiding variations in controller response due to singular events in the flow.

IMF stability was assessed by means of calculating RMS value for each IMF in each window. As visible in Figure 3, the stability of IMF for a short signal is low. Significant fluctuations of IMF RMS value are present, especially for the highest IMFs. It is even more evident when analyzing signals from instable operation regions. The values of IMF 1 to IMF 4 , which reflect the highest frequencies, are relatively stable. The stability for intermediate signals is much better, mostly very similar as stability for long signal. Therefore the IMF computed for 150000 samples was taken for further RMS analysis.

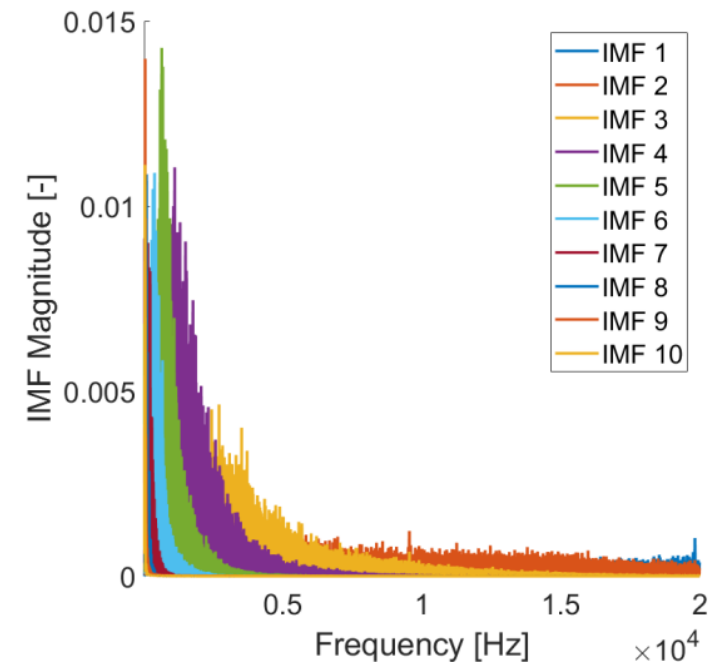

Figure 4. Fourier analysis of IMFs for $\mathbf{1 5 0} 000$ samples signal, TOA $=30 \%$

For a purpose of results interpretation, spectral analysis of IMFs was done for stable flow conditions $(\mathrm{TOA}=30 \%)$ at sensor upstream of the impeller $\left(p s-i m p_{1}\right)$. Fourier transform spectrum is shown in the figure 4 . The range of frequencies covered by each IMF in blower signal is presented. It is visible that the higher the IMF, the narrower frequency band it covers located at lower frequency. Therefore low frequency flow phenomena should be captured by highest IMFs, while high frequency variations, such as turbulence-induced noise ought to be contained in lowers IMFs.

\section{Indicators based on IMF energy}

Using EMD and assessing only IMF amplitude, it was possible to detect flow instabilities for a centrifugal blower. Varying power held by different IMFs is useful in detecting surge, as a global phenomenon and inlet recirculation, as a local one. Figures from 5 to 9 present RMS value for all IMF at varying TOA, plotted with solid lines, with scale on left vertical axis. Right vertical axis is connected with dashed line presenting the sum of all IMFs. Data for analysis was taken from one sampling window, having 150000 samples.

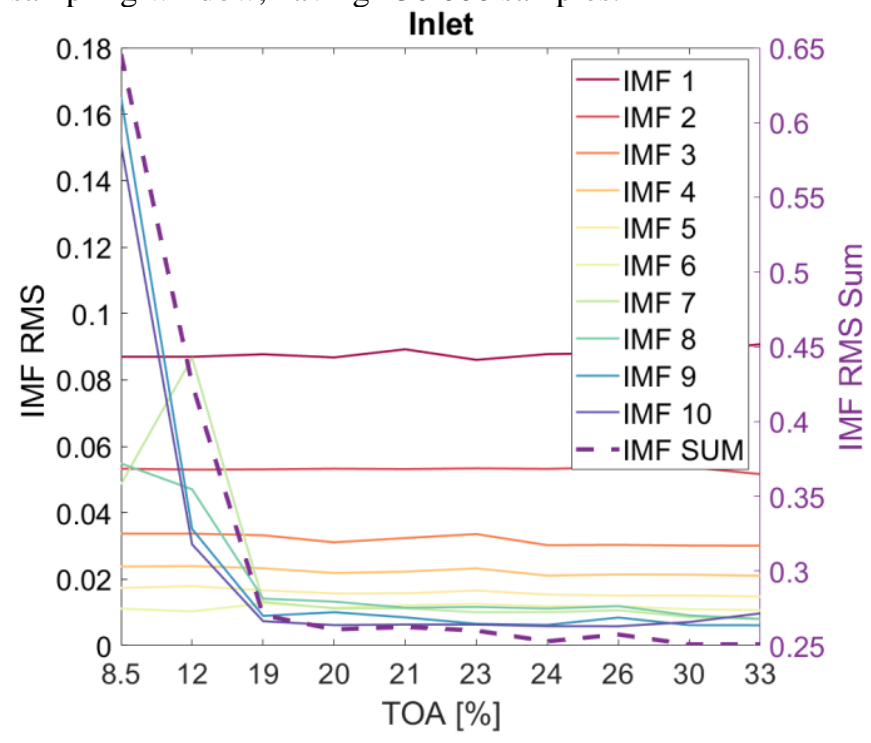

\section{Figure 5. IMF RMS values for inlet sensor ( $p s_{\text {in }}$ )}

In the figures, it is visible that there are global tendencies in IMF behavior. First IMF representing the highest frequency components is stable for every sensor location and TOA, being of the highest magnitude for all sensors in stable and transient conditions. Increase in IMFs 9 and 10, representing the lowest frequencies is visible for all sensors in the surge region. It is consistent with findings of $\mathrm{Wu}$ (Wu et al. 2016), who used amplitude of IMF as an indicator of the surge. They noted that the surge can be detected by an increase in an amplitude for one of the IMFs. In their case lower function (IMF 6) was used, yet due to lower sampling frequency the total number of intrinsic mode function was probably lower and the lowest frequencies could be captured by that IMF.

Inlet sensor (Figure 5) does not demonstrate important changes in any region, except for the surge onset. IMFs from 7 to 10 increase at the surge onset. The level of RMS is similar at the inlet and outlet for stable conditions. An increase in high IMFs is more significant for outlet than for inlet. The sum of IMF RMS fluctuates slightly at the region where inlet recirculation is present. Its important increase is visible for unstable conditions.

Before impeller (Fig. 6) the strongest influence of inlet recirculation is observable. All IMFs and their sum remain constant for stable conditions (30 and 33\% of TOA) and start to increase when approaching the zone of inlet recirculation. 
The peak of RMS is visible at $23 \%$ of TOA, going down until surge starts and going back up. The increase of RMS sum in that region is mainly due to IMFs 4 and 5. Further towards surge conditions, they decrease, influencing the sum. The inlet recirculation symptoms, probably being present up to mild surge occurrence (TOA 12\%) are, therefore, shared in between two IMFs. It might be due to the fact that the character of the recirculation changes, thus it has a different signature in terms of IMFs. Those observations incentivize further analysis aiming at correlating particular forms of phenomenon to each IMF.

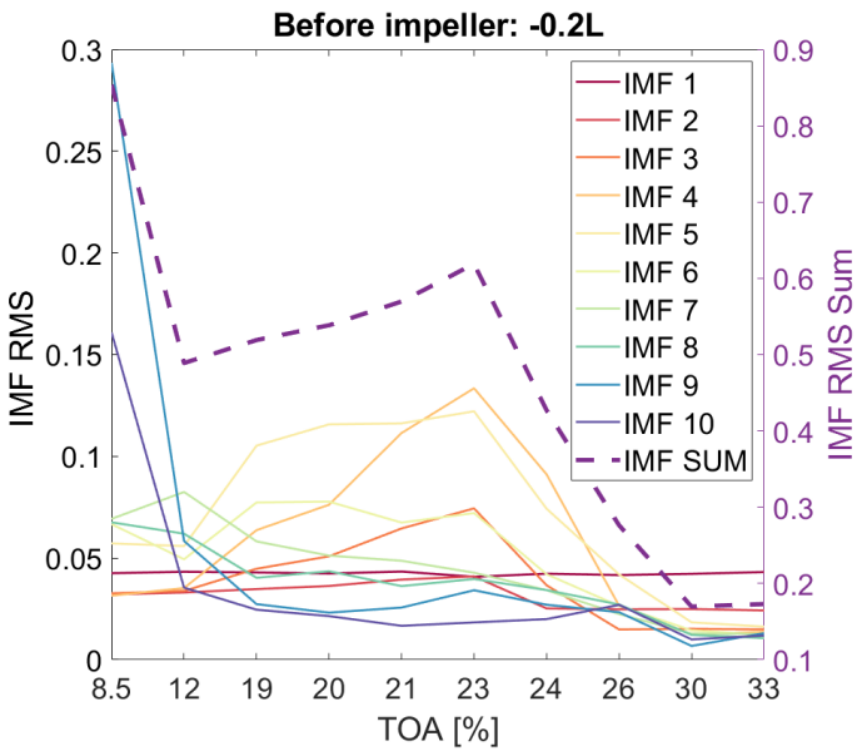

Figure 6. IMF RMS values for sensor ps $_{\text {imp1 }}$

The detection of inlet recirculation was a research subject for Logan et al. (Logan et al. 2018), who analyzed the same centrifugal blower. In their research, Singular Spectrum Analysis (SSA) method was capable of detecting a component indicating inlet recirculation when changing the TOA value from $26 \%$ to $24 \%$. The results obtained with EMD provide similar conclusions. Surge for this sensor is accompanied by a jump in IMF 9 and 10 value, as for most locations.

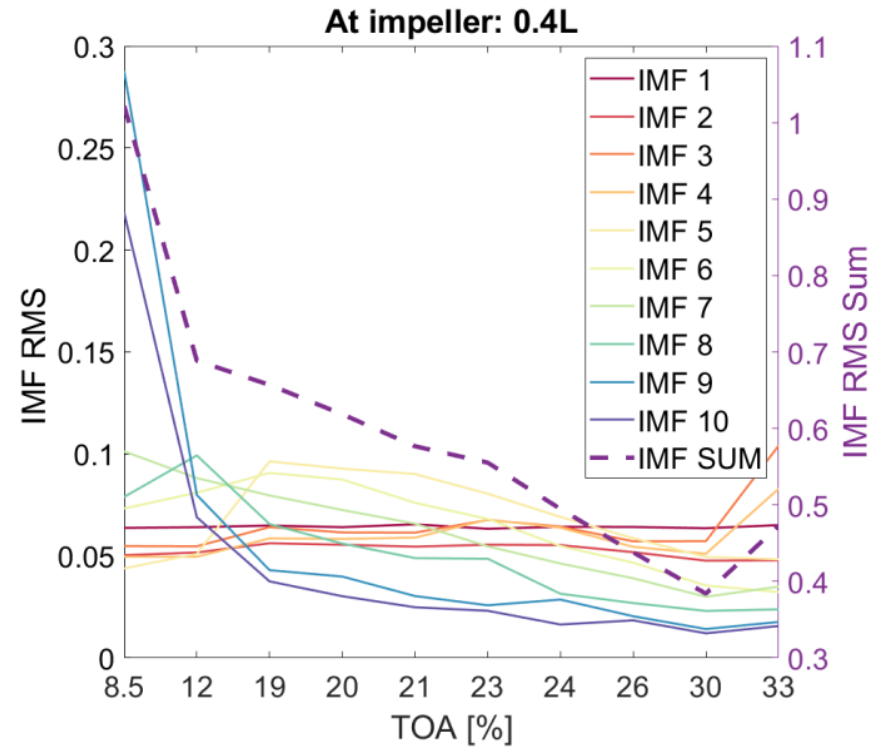

Figure 7. IMF RMS values for sensor ps imp2
At impeller (Fig. 7) some reflection of conditions before impeller are visible. RMS value of IMFs 3 and 4 is high for TOA $33 \%$, decreasing when reaching the optimum operation point (TOA $30 \%$ ). It is even more visible on RMS sum. This may suggest that optimum operation conditions can also be detected using EMD. IMF 5 tends to increase when approaching recirculation zone, but drops down at surge, similar behavior may be observed for IMF 6 .

An increase in IMF RMS value for inlet recirculation is not as significant as in case of sensor before impeller. IMF 7 and 8 increase from stable to unstable conditions, but no important change as for IMF 9 and 10 is visible when surge occurs.

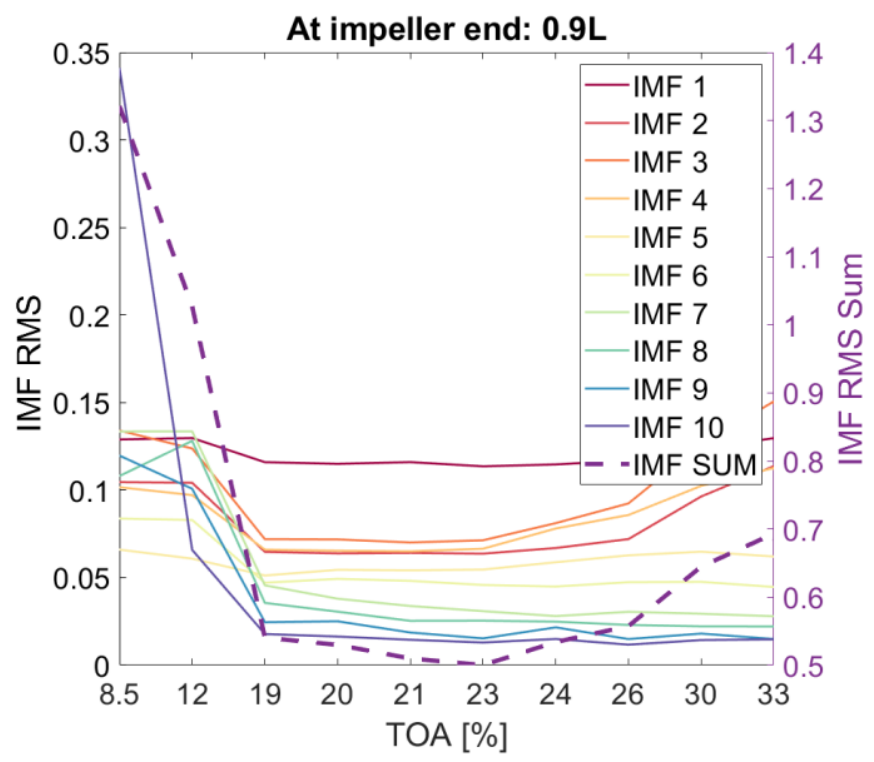

Figure 8. IMF RMS values for sensor $\mathbf{p s}_{\text {imp3 }}$

Different IMFs behavior is visible for impeller end (Fig. 8 ), where in stable conditions sum of IMF RMS is higher than for transient region. It is mostly due to IMFs 2,3 and 4 influence. It might be correlated with changes in flow structure, most likely tip vortices.

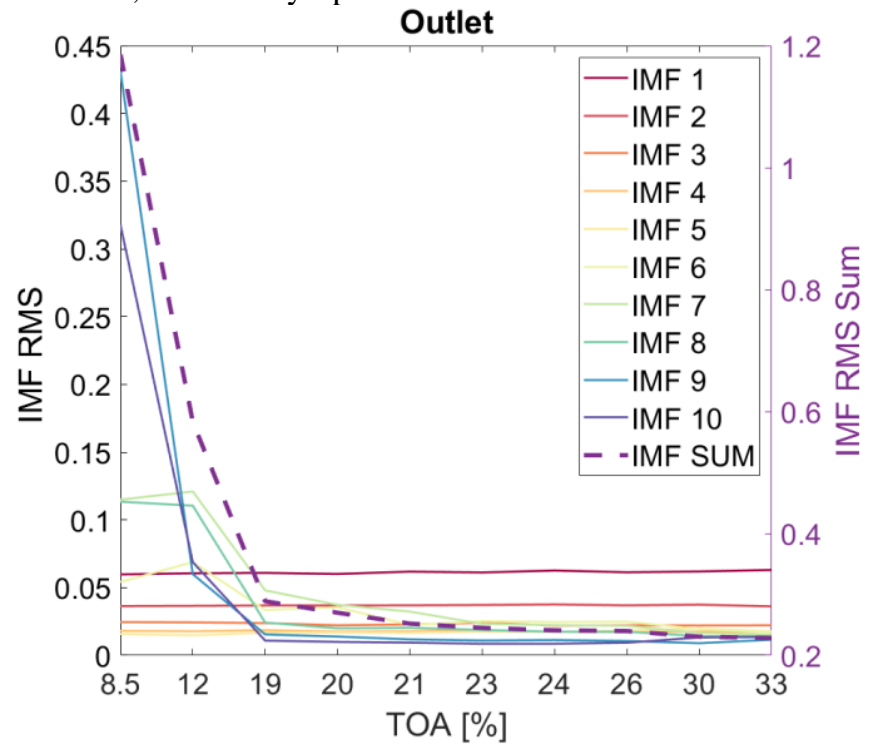

Figure 9. IMF RMS values for sensor at the outlet (ps out) 
The minimum of RMS energy is visible in the region of inlet recirculation. A further investigation of that aspect is necessary, yet it could be a potential method for long-time maintenance and detection of operating range with smaller tip leakage. Unlike for other sensors, a jump even in low IMFs is visible when entering the mild surge. It is also characteristic for this sensor that only IMF 10 grows further in the deep surge, with IMF 9 remaining at the same level as for the mild surge, unlike for other sensors.

The data obtained for outlet (Fig. 9) presents a similar behavior as inlet. An evident change in IMF RMS character is visible only for the surge conditions. Overall RMS value is similar to that at the inlet, yet for the surge it grows much higher than for the inlet. This is in line with finding of Liśkiewicz (Liskiewicz and Horodko 2015)

Empirical mode decomposition allows for obtaining intrinsic mode functions, which can bring more understanding of the analyzed signal. In literature, there is a number of methods which were applied to detect characteristic features. Most of them, however, was created to cater for bearings and gears research (Fan and Zuo 2008; Žvokelj et al. 2010). In that field, the faults are connected with mechanical damage and occur regularly with rotational frequency or its harmonics. The fault indicator have a short, intensive character, as for example increased vibration due to bearing race wear or acoustic emission due to cracks in a gear tooth. Thus, the goal of some methods is to amplify those events in the IMFs. The signals from turbomachinery are of different character and amplification of already strong events may lead to high exposure of blade passing frequency, rather than instabilities. Nevertheless, the richness of IMF analysis methods provokes further investigation of possible instabilities detection techniques in turbomachinery.

EMD, being proposed over 20 years ago has been modified, leading to creation of ensemble empirical mode decomposition (EEMD) and complete ensemble empirical mode decomposition (CEEMD). Those methods, assisted by added noise are proven to provide better decomposition (Lei et al. 2009; Yeh et al. 2010). The research in this paper have shown that even using standard EMD technique, it is possible to diagnose machine operation mode. Further research with use of EEMD and CEEMD would bring understanding about the potential benefits of those augmented methods.

\section{CONCLUSIONS}

In this article, the applicability of empirical mode decomposition for detection of flow instabilities in centrifugal blower was investigated. Static pressure measurements were subjected to EMD and analyzed.

Analysis of intrinsic mode functions RMS value allowed for detection of the surge and inlet recirculation in the considered data. IMF RMS value also presented interesting trend, decreasing at machine design conditions. It may present an opportunity for finding optimal working conditions, yet further investigation of this aspect is necessary.

The variability of IMFs in short time periods may be a valuable source of information about the flow, as short decomposition window tend to show higher IMF RMS fluctuations than longer sampling periods.

There exist other methods of IMF analysis, which could give different insights about the signal, thus character of the flow in a blower or other rotating machinery. Due to the signal origin and expected form, the energy-based approach applied in this article seems the most suited for instabilities detection using pressure sensor data.

\section{ACKNOWLEDGMENTS}

This work was funded by the National Centre for Research and Development (LIDER/447/L6/NCBR/2015).

\section{BIBLIOGRAPHY}

Bin GF, Gao JJ, Li XJ, Dhillon BS (2012) Early fault diagnosis of rotating machinery based on wavelet packets - Empirical mode decomposition feature extraction and neural network. Mech Syst Signal Process 27:696-711. doi: 10.1016/j.ymssp.2011.08.002

Day IJ (2015) Stall, Surge, and 75 Years of Research. J Turbomach 138:011001. doi: 10.1115/1.4031473

Fan X, Zuo MJ (2008) Machine fault feature extraction based on intrinsic mode functions. Meas Sci Technol 19:. doi: 10.1088/0957-0233/19/4/045105

Fink DA, Cumpsty NA, Greitzer EM (2008) Surge Dynamics in a Free-Spool Centrifugal Compressor System. J Turbomach 114:321. doi: 10.1115/1.2929146

Garcia D, Liśkiewicz G (2016) Stable or not stable? Recognizing surge based on the pressure signal. Trans Inst Fluid-Flow Mach 133:55-68

Garcia D, Stickland M, Lis̈kiewicz G (2015) Dynamical system analysis of unstable flow phenomena in centrifugal blower. Open Eng 5:332-342. doi: 10.1515/eng-2015-0036

Goharrizi AY, Sepehri N (2012) Internal leakage detection in hydraulic actuators using empirical mode decomposition and hilbert spectrum. IEEE Trans Instrum Meas 61:368-378. doi: 10.1109/TIM.2011.2161938

Huang NE (2014) Hilbert Huang Transform and its applications. World Scientific

Huang NE, Shen Z, Long SR, et al (1998) The empirical mode decomposition and the Hilbert spectrum for nonlinear and non-stationary time series analysis. doi: 10.1098/rspa.1998.0193

Kurz R, Zwerver R (2018) Dynamic simulation and testing to assess rundown speed of a compressor. Director 15:2017-2019.

doi: 10.22201/fq.18708404e.2004.3.66178

Lei Y, He Z, Zi Y (2009) Application of the EEMD method to rotor fault diagnosis of rotating machinery. Mech Syst Signal Process 23:1327-1338. doi: 10.1016/j.ymssp.2008.11.005

Lei Y, Lin J, He Z, Zuo MJ (2013) A review on empirical mode decomposition in fault diagnosis of rotating machinery. Mech Syst Signal Process 35:108-126. doi: 10.1016/j.ymssp.2012.09.015

Li C, Lei Y, Fu R (2011) Aerodynamic instability detection in 
compressor based on Hilbert-Huang transform. Proc 2011 IEEE Int Conf Comput Sci Autom Eng CSAE 2011 4:355-358. doi: 10.1109/CSAE.2011.5952867

Li H, Zhang Y, Zheng H (2009) Hilbert-Huang transform and marginal spectrum for detection and diagnosis of localized defects in roller bearings. J Mech Sci Technol 23:291-301. doi: 10.1007/s12206-008-1110-5

Liśkiewicz G (2014) Numerical Model of the Flow Phenomena Preceding Surge in the Centrifugal Blower and Assessment of Its Aplicability in Designing Antisurge Devices, $\mathrm{PhD}$ thesis. University of Strathclyde

Liskiewicz G, Horodko L (2015) Time-frequency analysis of the Surge Onset in the Centrifugal Blower. Open Eng 5:299-306. doi: 10.1515/eng-2015-0040

Liśkiewicz G, Horodko L, Stickland M, Kryłłowicz W (2014) Identification of phenomena preceding blower surge by means of pressure spectral maps. Exp Therm Fluid Sci 54:267-278. doi: 10.1016/j.expthermflusci.2014.01.002

Logan A, Garcia D, Liśkiewicz G (2018) Detection of inlet recirculation as an early indicator of proximity to instability in centrifugal compressors . 13th Int Symp Compress Turbine Flow Syst - Theory Appl Areas

Singh DS, Zhao Q (2016) Pseudo-fault signal assisted EMD for fault detection and isolation in rotating machines. Mech Syst Signal Process 81:202-218. doi: 10.1016/j.ymssp.2016.03.007

Wu X, Liu Y, Liu R, Zhao L (2016) Surge detection methods using empirical mode decomposition and continuous wavelet transform for a centrifugal compressor. J Mech Sci Technol 30:1533-1536. doi: 10.1007/s12206-0160307-2

Yeh J-R, Shieh J-S, Huang NE (2010) Complementary Ensemble Empirical Mode Decomposition: a Novel Noise Enhanced Data Analysis Method. Adv Adapt Data Anal 02:135-156. doi: 10.1142/s1793536910000422

Yu D, Cheng J, Yang Y (2005) Application of EMD method and Hilbert spectrum to the fault diagnosis of roller bearings. Mech Syst Signal Process 19:259-270. doi: 10.1016/S0888-3270(03)00099-2

Žvokelj M, Zupan S, Prebil I (2010) Multivariate and multiscale monitoring of large-size low-speed bearings using Ensemble Empirical Mode Decomposition method combined with Principal Component Analysis. Mech Syst Signal Process 24:1049-1067. doi: 10.1016/j.ymssp.2009.09.002 